\title{
Instabilities of conducting fluid layers in weak time-dependent magnetic fields
}

\author{
I. Cortés-Domínguez and J. Burguete* \\ Departamento de Física y Matemática Aplicada, Facultad de Ciencias, Universidad de Navarra, Navarra, Spain
}

(Received 15 February 2017; published 6 July 2017)

\begin{abstract}
We present the experimental analysis of the instabilities generated on a large drop of liquid metal by a time-dependent magnetic field. The study is done exploring the range of tiny values of the control parameter (the ratio between the Lorentz forces and inertia) avoiding nonlinear effects. Two different instabilities break the symmetries generating spatial patterns that appear without a threshold for some specific frequencies (up to the experimental precision) and have been observed for parameter values two orders of magnitude lower than in previously published experiments [J. Fluid Mech. 239, 383 (1992)]. One of the instabilities corresponds to a boundary condition oscillation that generates surface waves and breaks the azimuthal symmetry. The other corresponds to a parametric forcing through a modulation of the Lorentz force. The competition between these two mechanisms produces time-dependent patterns near codimension- 2 points.
\end{abstract}

DOI: 10.1103/PhysRevE.96.013103

\section{INTRODUCTION}

The existence of nonlinear effects and instabilities is a key point on the dynamics of many natural, industrial, and even social processes [1]. Despite all the effort that has been done on extended systems there is a lack of understanding of small problems, where the spatial dimensions are comparable to any characteristic length produced by the instability. Many different configurations have been analyzed: small containers shaken by vertical vibration [2], droplets driven by ultrasounds [3], small convective problems [4,5], colloidal droplets with electrical fields [6], or liquid metals with electric [7] or magnetic [8-15] fields.

This last configuration is a particular case of a discipline, magnetohydrodynamics (MHD) [16], that studies the interaction between conducting fluids and magnetic fields and that is involved in a broad variety of fields: biophysics [17], crystal growth [18], metallurgy [19], heat transfer [20], geo- and astrophysics [21], plasma physics [22], and electrolysis [23]. A common issue in these problems is that MHD instabilities are usually observed very far from a threshold because of the experimental difficulties in detecting weak effects. The consequence is that in many configurations the MHD forces are usually neglected [17].

These effects have been recently observed in humans [24]: a MHD mechanism has been proposed to explain the origin of vertigoes suffered by some individuals when they are placed inside an MRI device. The authors propose that pressure fluctuations are produced in the electrolyte present in the inner ear by the interaction of ionic currents with the magnetic field. Other instabilities where no external currents are involved were neglected, although pure MHD instabilities without a threshold have been proposed but in a different context [10]. However, in this last case all the experiments up to now were done in a strongly nonlinear regime [8,9,11-15], a common feature of many of the examples cited above.

In this paper we analyze the different mechanisms that can trigger instabilities in a conducting fluid layer under

\footnotetext{
*javier@unav.es
}

the action of very weak time-dependent magnetic fields. The experimental setup allows us to explore regions with bifurcation parameters two orders of magnitude smaller than those of previous works [9]. The experimental study is based on the observation of surface modulations. We have observed symmetry breaking for tiny values of the bifurcation parameter and identified two different mechanisms involved in the pattern creation.

\section{EXPERIMENTAL SETUP}

The experimental cell consists of a cylindrical Teflon ${ }^{\circledR}$ cavity with an inner diameter of $\varnothing 84 \mathrm{~mm}$. The inner bottom is flat for $r \leq 20 \mathrm{~mm}$ and slightly inclined for $r>20 \mathrm{~mm}$ (Fig. 1). A volume $V_{d}$ of an eutectic InGaSn alloy, liquid at room temperature (density $\rho=6360 \mathrm{~kg} / \mathrm{m}^{3}$, electrical conductivity $\sigma=3.110^{6} \mathrm{~S} / \mathrm{m}$, interfacial tension $\gamma=0.53 \mathrm{~N} / \mathrm{m}$ ) [25], is placed inside and remains centered because of the depression. Due to the surface tension [25] the liquid metal adopts the shape of a thick circular fluid drop. This drop is immersed in a $\mathrm{HCl} 1 \%$ water dilution to prevent oxidation. The whole setup is closed by a transparent cover. For all the results presented in this paper the working volume is $28 \mathrm{~cm}^{3}$ with an external radius of $R_{d}=35 \mathrm{~mm}$ and a thickness at the center $h_{d}=11 \mathrm{~mm}$. Only two cases are included with a volume of $V_{d}=35 \mathrm{~cm}^{3}$ that correspond to videos in the Supplemental Material.

The magnetic field is generated by an alternating electrical current $I_{c}$ in an external coil, with a frequency between 0.1 and $10 \mathrm{~Hz}$ and an intensity up to $60 \mathrm{~A}$. This range of frequencies is small enough to prevent skin effects, so the axisymmetric magnetic field fully penetrates the fluid drop. The vertical component is uniform in the vertical ( $z$ axis) direction (with variations below $0.2 \%$ ) and increases approaching the lateral wall (up to $7 \%$ stronger). The radial component increases almost linearly with the radius, up to $3 \%$ of the maximum value of the vertical component, so we can assume that we are forcing with a nearly vertical magnetic field. We can then conclude that the field evolves harmonically: $\vec{B}_{e}=B_{0} \sin \left(2 \pi f_{B} t\right) \hat{k}$, with $f_{B}=\omega_{B} / 2 \pi$ the forcing frequency and $B_{0}$ the amplitude. For the maximum current $I_{c}=60 \mathrm{~A}$ the corresponding magnetic field amplitude is $B_{0}=0.07 \mathrm{~T}$. 


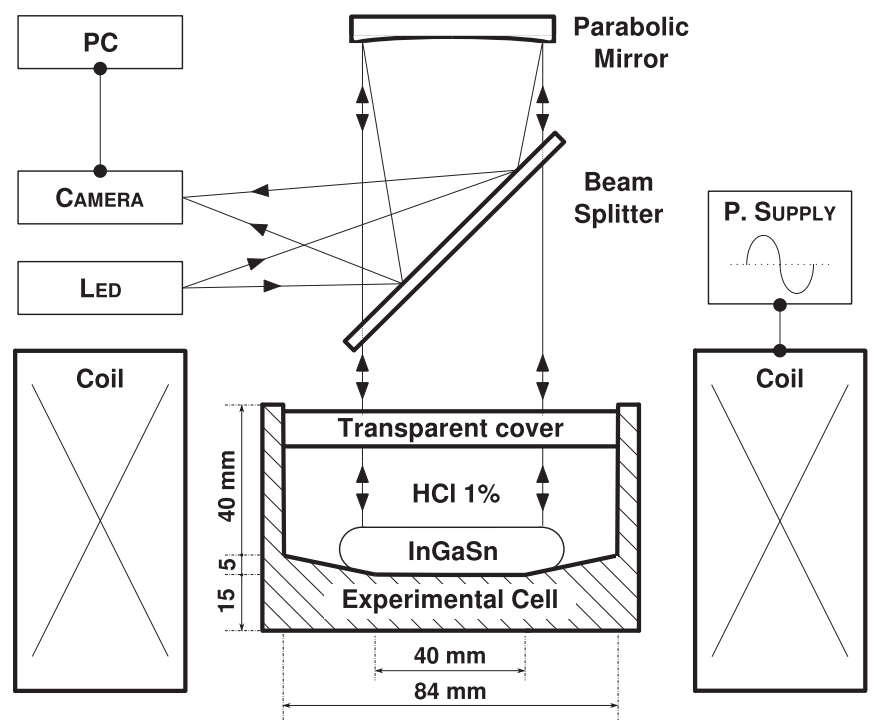

FIG. 1. Cross section of the experimental cell (size in mm) and diagram of the experimental setup (not to scale).

Let us summarize the key points of the mechanism that triggers the instabilities: The temporal evolution of the magnetic field produces magnetic flux density variations giving rise to a current density $\vec{j}$ due to the Lenz's law: when the external magnetic field $B_{e}$ changes, an eddy current is created to compensate for this variation producing an induced magnetic field $b_{i}$ [16]. The induced currents interact again with the magnetic field, so a Lorentz force $\vec{j} \times \vec{B}$ appears, where $\vec{B}=\vec{B}_{e}+\vec{b}_{i}$ accounts for the external $\vec{B}_{e}$ and induced $\vec{b}_{i}$ components. This process is valid in the magnetohydrodynamic approximation, which states that the evolution of $\vec{B}$ must be slow and the conductor remains free of charge. This new force can destabilize the liquid drop in different ways.

One possibility that becomes experimentally evident for large magnetic fields is the axisymmetric drop beating. When the magnetic field is reduced (respectively, increased), the radial Lorentz force increase (reduces) the radius $R_{d}$ of the drop in order to keep constant the magnetic flux. This mechanism forces the beating of the drop.

The relevance of the Lorentz force compared to inertia is expressed by the interaction parameter $N=B_{0}^{2} L \sigma / \rho U$ [16] where $B_{0}$ is the amplitude of the forcing magnetic field, $L$ is a characteristic length (typically, the droplet radius), and $\sigma$ and $\rho$ the electrical conductivity and density of the fluid, respectively. The characteristic velocity $U$ of the flow is obtained from the beating of the drop and can be estimated as $U=2 \pi f_{B} L=$ $L \omega_{B}$, so the interaction parameter becomes $N=B_{0}^{2} \sigma / \rho \omega_{B}$ [10].

We have determined the presence of instabilities in our experiments down to a minimum value of $N_{\min }=0.0003$. For comparison, the interaction of the MRI device with the inner ear will produce a $N \sim 0.002 \omega_{B}^{-1}$ for a magnetic field of $B \sim 3 \mathrm{~T}$ interacting with either the endolymph (basically, a $\mathrm{KCl}$ solution) or perilymph ( $\mathrm{NaCl}$ dilution) [26]. Other experimental setups have reached a minimum of $N \gtrsim 0.02$, nearly two orders of magnitude larger than the values attained in this work.

The optical system consists of a shadowgraphy [27] but with a reflecting curved surface (the liquid metal surface) instead of a phase object: a punctual light source with controlled illumination power is placed at the focus of a parabolic mirror. A parallel beam is generated with vertical incidence to the water-InGaSn interface, where it is reflected. Instabilities may create surface deflections that deviate the light and induce intensity modulations. A camera with fixed gain located on the focus of the reflected beam records the temporal evolution of the pattern. Due to this setup, only the central part $(r<29 \mathrm{~mm})$ of the droplet can be observed. The recovered intensities allow a direct comparison between the dynamics in different regions of the phase space. This setup has already been used in other experiments [28] and in the present configuration can measure modulations of the surface peak-to-peak of $10 \mu \mathrm{m}$. The lateral resolution is $0.3 \mathrm{~mm}$. The light intensity $I$ scaled between the bright and dark regions allows us to study the dynamics of the instabilities and reconstruct the patterns.

\section{RESULTS}

Because of the forcing mechanism responsible of the instabilities, all the patterns observed here have a dynamics governed by the oscillation frequency of the Lorentz term of the Navier-Stokes equation $\vec{j} \times \vec{B}$, which is twice the excitation frequency $f_{L}=2 f_{B}=\omega_{B} / \pi$.

In Fig. 2 we present a subset of all the different patterns that can be recovered (see videos of the dynamical behavior in Ref. [29]). The first patterns that appear without threshold are axisymmetric:, $I(x, y, t)=I(r, t)$. When the forcing strength is increased (for larger values of $\vec{B}_{e}$ ), these patterns break the axisymmetry $I(r, \theta, t)=I(r, t) \exp (i m \theta)$. All the patterns that have been observed correspond to patterns with an azimuthal wave number $m=2$ to 10 . For the drop size considered here we only clearly detect azimuthal wave numbers, although some modulations on the radial direction are visible.

In Fig. 3 we present the phase space of these patterns. Each pattern is split into axisymmetric and nonaxisymmetric parts $I(r, \theta, t)=I_{A x}(r, t)+I_{\mathrm{NoAx}}(r, \theta, t)$. We have performed a Fourier expansion of the temporal evolution for the last term of the previous expression $I_{\mathrm{NoAx}}(r, \theta, t)=$ $\sum_{n=0}^{\infty} A_{n}(r, \theta) \exp \left(i n \omega_{B} t\right)$. We have determined that a nonaxisymmetric pattern was triggered when their weight $W_{\mathrm{NoAx}}=$ $\sum_{n=1}^{\infty} \int_{S} A_{n}^{2} d s$, compared to the maximum value, was larger than a threshold value $W_{\mathrm{Th}}=W_{\mathrm{NoAx}} / W_{\mathrm{Max}}$ comparable to the noise level of the acquisition system.

With this idea in mind, we have used a threshold value of $W_{\mathrm{Th}}^{\text {even }}=0.06$ for Figs. 3(a), 3(b), and 3(d) and $W_{\mathrm{Th}}^{\text {odd }}=0.02$ for Fig. 3(c). Although these values are to some extent arbitrary, they allow us to distinguish axisymmetric and nonaxisymmetric regions of the phase space separated by a boundary $B_{\text {Thr }}\left(f_{b}\right)$. In all the panels, the gray regions correspond to the axisymmetric patterns (i.e., the nonaxisymmetric part remains below the noise level). The threshold boundary $B_{\mathrm{Thr}}\left(f_{B}\right)$ for the whole nonaxisymmetric part has local minima on $f_{B}=1.8$, 2.9, 4.1, and $5.4 \mathrm{~Hz}$. These minima present nonaxisymmetric behavior even for the minimum value of the magnetic field 

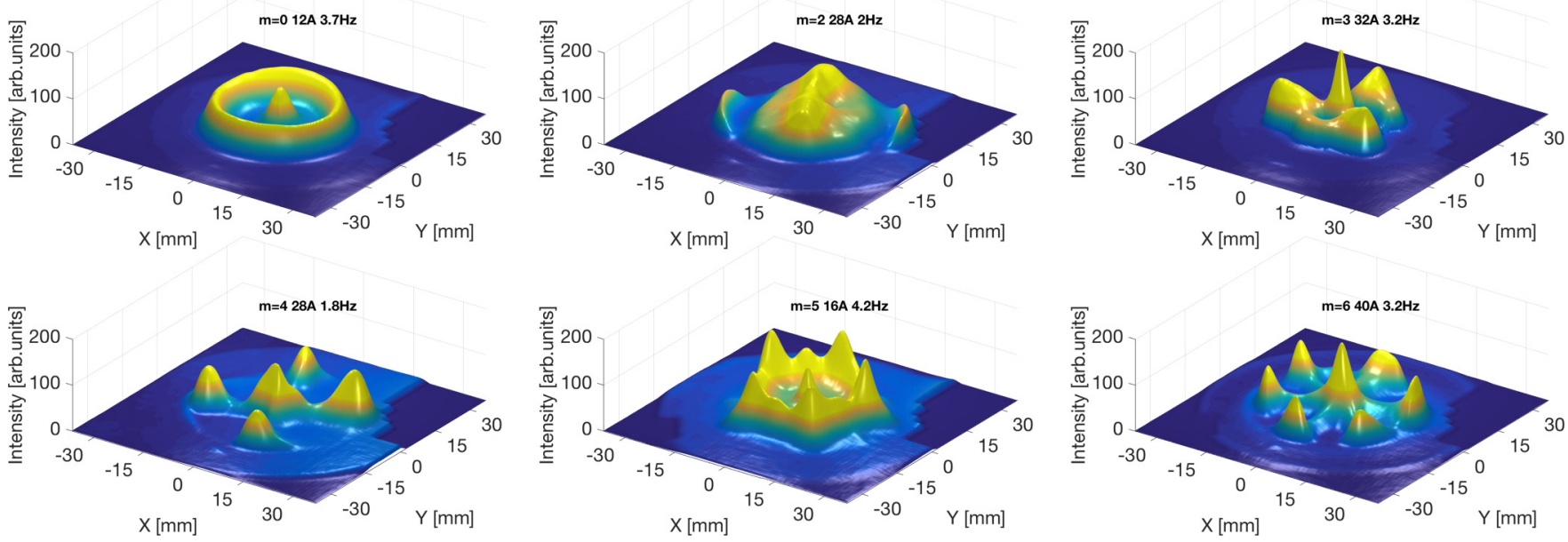

FIG. 2. Light intensity $I$ recovered using the observational setup from the surface modulation across the horizontal plane (in pixels). From left to right, top to bottom, patterns with a dominant azimuthal wave number $m=0,2,3,4,5,6$. The excitation frequency $f_{e}$ and the intensity current are indicated. Mode $m=0$ corresponds to a pattern with a very weak nonaxisymmetric component (see fig. 3). Other patterns (not plotted here) obtained for $m=8$ and 10 and movies of the different dynamics are presented as Supplemental Material[29]. Only the central $r<29 \mathrm{~mm}$ of the drop are visible (see details in the text).

that we can attain in our setup. For this reason, we consider that for these frequencies there is no excitation threshold. No hysteresis has been detected.

In Fig. 3(a) we present a simplified phase space covering the observed patterns. Each symbol represents a different azimuthal symmetry. When a single azimuthal wave number has been detected, only one symbol appears. When the threshold has been crossed, but the data do not allow us to differentiate between the various possible azimuthal wave numbers, we have indicated those cases with a circle. In other cases, different wave numbers were detected in the dynamical cycle of the pattern for the same values of the experimental parameters. Two possibilities arise. The first one consists of a cycle between patterns with different $m_{i}$ for each time period, i.e., the azimuthal wave number is time-dependent $m(t)$ or, alternatively, the amplitude of each pattern $m_{i}$ oscillates. In those situations the wave numbers present are indicated in Fig. 3(a) separated by a slash. In some special cases a more complex dynamics between three modes can be obtained (not shown here). When only one of the wave numbers is clearly identified, but not the other, the dynamical behavior is indicated with a solid marker. In Fig. 3(b) we include all the experimental parameters so the correspondence between $I_{c} \leftrightarrow B \leftrightarrow N$ can be derived to allow an easier interpretation of the other panels.

As pointed out above, the forcing frequency in the Lorentz term of the Navier-Stokes equation corresponds to $f_{L}=2 f_{B}$. To differentiate between the possible mechanisms, we have split the temporal evolution onto the even and odd multiples of the magnetic field frequency $f_{B}$ [see Figs. 3(c) and 3(d)], i.e., harmonics and subharmonics of the forcing frequency $f_{L}$. We can observe a very different behavior for each panel, with different thresholds. The even modes [Fig. 3(d)] correspond to harmonics of the frequency $f_{L}=2 f_{B}$, and the instability appears without threshold for some specific frequencies. On the other side, the odd modes correspond to subharmonics of the frequency of the Lorentz force, and in this case the instability appears for nonzero values of the interaction parameter $N_{\text {Th }}$.

All these waves correspond to surface waves and should obey the dispersion relation:

$$
\omega_{n}^{2}=\left[\frac{\rho_{M}-\rho_{D}}{\rho_{M}+\rho_{D}} g k+\frac{\gamma}{\rho_{M}-\rho_{D}} k^{3}\right] \tanh (k h),
$$

where $g, \gamma, \rho_{M}$, and $\rho_{D}$ correspond to gravity, surface tension (see Ref. [13]), and densities of the InGaSn alloy and the $\mathrm{HCl}$ water dilution, respectively. In Refs. [30-32] the MHD effects are considered, including corrections to the linear and cubic terms. Nevertheless in our case the magnetic damping time $\tau=\rho / \sigma B_{0}^{2} \sim 1000 \mathrm{~s}$ [32] is so large that these modifications of the dispersion relation are negligible.

The frequencies where the minimum threshold is obtained are $f_{L}^{\min }=1.8,2.9$, and $4.1 \mathrm{~Hz}$ for the even modes and $f_{L}^{\text {min }}=$ $3.4,4.5$, and $5.8 \mathrm{~Hz}$ for the odd modes. These modes are plotted in Fig. 4.

The behavior of the even modes corresponds to the breaking of the axisymmetry for the beating of the drop, which excites surface waves with the same frequency of the forcing mechanism. Here, instead of a vertical (or lateral) shaking of the fluid layer, the destabilizing force is the radial pulsation of the droplet induced by the Lenz currents. The observed frequencies correspond to multiples of the forcing frequency $\omega_{n}^{\text {even }}=\omega_{L}=2 \omega_{B}$.

We present the wave number of the observed patterns for the even modes (Fig. 4, *), associated to the first $m=$ 2-5 azimuthal modes $\lambda_{m}=2 \pi R / m$ and their corresponding frequencies. A good agreement with Eq. (1) is observed (solid line). As the capillary length is very small $l_{c}=4 \mathrm{~mm}$ and for the wave numbers considered here, we can consider that we are on the shallow water regime $\left(k H \lesssim 1 ; k l_{c} \ll 1\right)$ so $\omega_{n} \sim k \sqrt{g H\left(\rho_{M}-\rho_{D}\right) /\left(\rho_{M}+\rho_{D}\right)}$. 

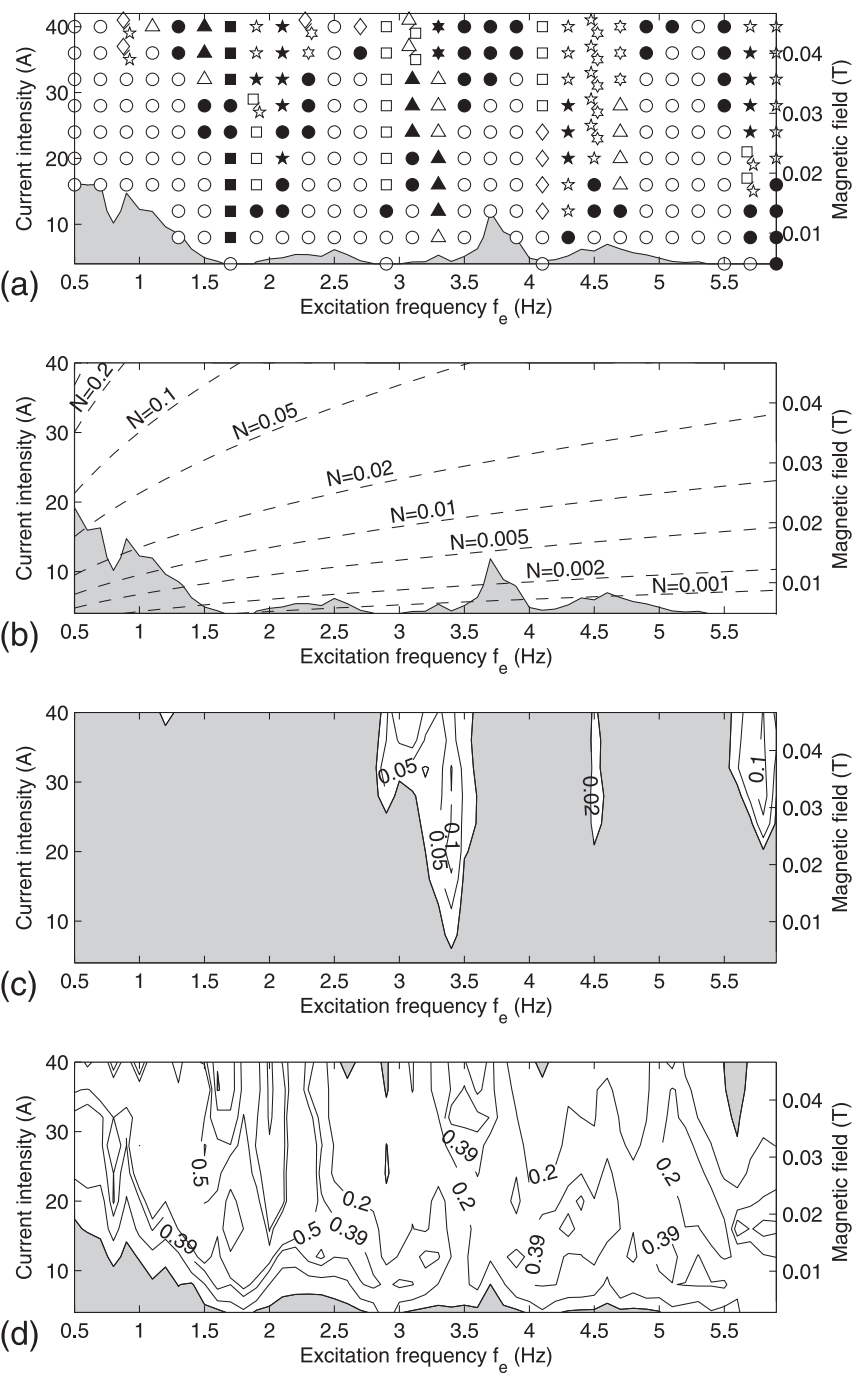

FIG. 3. Phase space of the instabilities. (a) Observed patterns: $m=2, \diamond ; m=3, \Delta ; m=4, \square ; m=5, \star ; m=6, \star$; other nonaxisymmetric patterns, $\circ$. When a mixed state is observed, the observed patterns appear separated by a slash. Void symbols mean a stable pattern, full symbols a state for dynamical cases. (b) Threshold for the instabilities based on the cumulative energy for the whole set of observed modes for the nonaxisymmetric part. The different axes denote the values of the variables $f_{e}, I_{c}, B$, and $N$. Cumulative energy for the (c) odd and (d) even modes, referred to as the excitation frequency $f_{B}$. The gray zones correspond to regions where the cumulative energy is smaller than the threshold value $W_{\text {Th }}$ (see text for explanation).

The oscillations observed for the odd modes correspond to the behavior predicted by Ref. [10] that were observed in Ref. [9] but in a strongly nonlinear regime and far from the threshold. The corresponding eigenfunctions excited on the surface can be described as $\mathrm{e}^{i m \theta} J_{m}\left(k_{m n} r\right)$ where $m$ is the azimuthal wave number and $k_{m n}$ is the $n$th zero of the function $J_{m}\left(k_{m n} R\right)$. The evolution of this modes obeys, to the first order, a Mathieu-Hill equation [10]:

$$
\ddot{a}_{n}+\omega_{n}^{2}\left[1+2 \epsilon \sin \left(\omega_{L} t\right)\right] a_{n}=0
$$

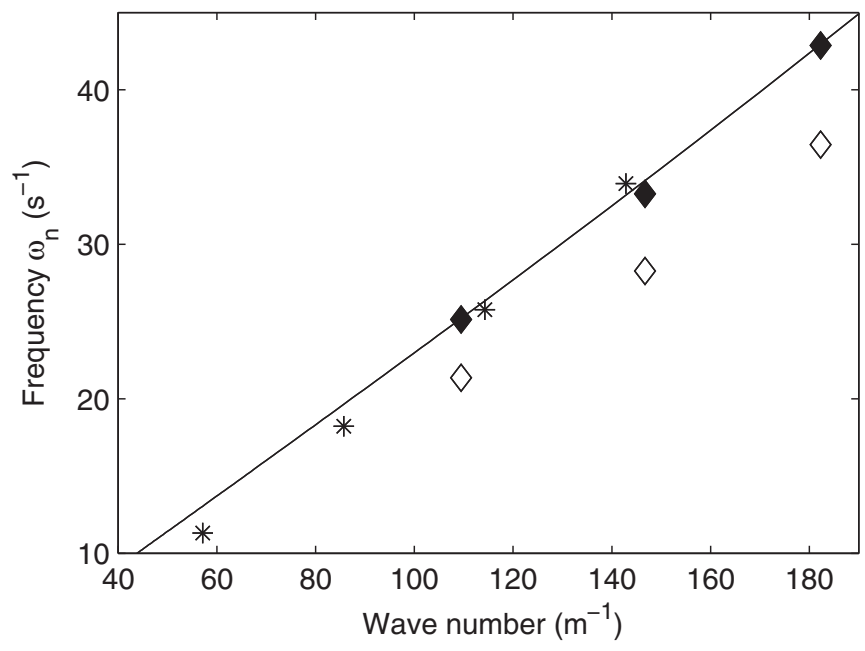

FIG. 4. Dispersion relation observed for the excited waves at the minima observed of the threshold boundary $B_{\mathrm{Thr}}\left(f_{B}\right): *$, even modes (laterally excited); $\diamond$, odd modes (MHD instability) without lateral damping (open) and with damping (closed). The solid line corresponds to the dispersion relation for surface waves (no adjustable parameters).

where $a_{n}$ represents the amplitude of the different waves with frequencies $\omega_{n}$. This equation reproduces the response of many different parametrically forced systems [33], and it can resonate with subharmonics of the excitation frequency. In this case the first odd mode corresponds to $\omega_{n}=\omega_{L} / 2$.

For the odd modes, the wave numbers are the solution of the previous Bessel functions. The modes with the lowest frequencies correspond to the first zeroes of $J_{m}\left(k_{m 1} R\right)$. In Fig. 4 we present the associated wave numbers $k_{m 1}$ for the first $m=1-3$ modes and the associated frequencies (open diamonds). We can note that there is a drift between the observed frequencies and the values obtained from the dispersion relation. Actually, the observed oscillation frequency is smaller than the expected behavior. As was already proposed in Ref. [34] the presence of a lateral meniscus can reduce the observed frequencies and affect the dynamical behavior of surface waves modifying the damping. The solid diamonds presented in Fig. 4 correspond to a correction of the observed frequencies assuming a 15\% reduction induced by the damping. This damping is also responsible of the nonzero value of the threshold.

The simultaneous existence of both mechanisms for the same parameter values in the phase space induces multistability. In these situations, we are close to codimension-2 points, and the patterns have a very complex dynamics where two or even three alternating patterns were observed. This is relevant around [regions with solid markers in Fig. 3(a)] the frequency intervals $1.5-2.5,3-3.5,4.5$, and 5.5-6.0 Hz. The last three ranges correspond to the regions with $m=1,2$, and 3 for the odd modes. Nevertheless, there is no odd mode identified on Fig. 3(c) for the first island $1.5-2.5 \mathrm{~Hz}$. This region will correspond to the mode $m=0$ that would have an associated frequency of $2.1 \mathrm{~Hz}$ according to the presented description, which lies just in the middle of the observed region. This mode cannot be observed here because, being axisymmetric, 
cannot be separated from the basic axisymmetric pulsation Nevertheless, its presence can be detected indirectly by the multistability region around $f=2 \mathrm{~Hz}$.

\section{CONCLUSIONS}

We have presented a study of an experimental setup where a fluid layer is destabilized by a time-dependent magnetic field. Different nonaxisymmetric patterns were obtained, and a detailed phase space has been presented. We have clearly identified two different mechanisms that produce different patterns with different dynamics. These two mechanisms generate a different response, with oscillations that can be harmonics or subharmonics of the forcing frequency. All the observed instabilities are recovered for a value of the bifurcation parameter two orders of magnitude lower than in previous experiments.

For the even modes (even multiples of $\omega_{B}$ ) different azimuthal waves are detected without threshold, or with a threshold smaller than the experimental resolution $(N<$ 0.0003). For the odd modes, we have determined that the threshold would correspond to an interaction parameter between $N=0.001$ and $N=0.01$ depending on the excited frequency. Moreover, the wave numbers and frequencies associated are different in each process. These values of the interaction parameter can be reached on MRI devices. Further work in this direction is needed to clarify if this mechanism can affect the vestibular cavity. Even more, these results open the door to the possibility of MHD instabilities in other configurations where the magnetic field effects are usually considered as too weak and neglected.

\section{ACKNOWLEDGMENTS}

We are grateful to W. González-Viñas for fruitful discussions. This work is supported by the Spanish Government through research projects FIS2011-24642 and FIS201454101-P. I.C.-D. acknowledges "Friends of the University of Navarra" for a grant.
[1] M. C. Cross and P. C. Hohenberg, Rev. Mod. Phys. 65, 851 (1993).

[2] J. Rajchenbach, D. Clamond, and A. Leroux, Phys. Rev. Lett. 110, 094502 (2013).

[3] C. L. Shen, W. J. Xie, and B. Wei, Phys. Rev. E 81, 046305 (2010).

[4] J. Burguete and H. Mancini, Eur. Phys. J. Special Topics 223, 9 (2014).

[5] I. Cortés-Domínguez, J. Burguete, and H. L. Mancini, Phil. Trans. R. Soc. A 373, 20150113 (2015).

[6] A. Varshney, S. Ghosh, S. Bhattacharya, and A. Yethiraj, Sci. Rep. 2, 738 (2012).

[7] E. Ramírez-Álvarez, J. L. Ocampo-Espindola, F. Montoya, F. Yousif, F. Vázquez, and M. Rivera, J. Phys. Chem. A 118, 10673 (2014).

[8] Y. Fautrelle, A. D. Sneyd, and J. Etay, Fluid Mechanics: Its Applications (Springer, Amsterdam, 2007), Vol. 80, pp. 345-355.

[9] J. M. Galpin and Y. Fautrelle, J. Fluid Mech. 239, 383 (1992).

[10] Y. Fautrelle and A. D. Sneyd, Eur. J. Mech. B. 24, 91 (2005).

[11] Y. Fautrelle, J. Etay, and S. Daugan, J. Fluid Mech. 527, 285 (2005).

[12] K. Spragg, A. Sneyd, and Y. Fautrelle, Magnetohydrodynamics 45, 543 (2009).

[13] Ch. Karcher, V. Kocourek, and D. Schulze, in Proceedings of the International Scientific Colloquium Modelling for Electromagnetic Processing, Hannover, March 24-26, 2003 (University of Hannover, Germany, 2003), pp. 105-110.

[14] D. Schulze, Ch. Karcher, V. Kocourek, and J. U. Mohring, in Proceedings of the 4th International Scientific Colloquim 'Modelling for Material Processing, Riga, June 8-9, 2006 (University of Latvia, Laboratory for Mathematical Modelling, of Environmental and Technological Processes, Riga, Latvia, 2006).

[15] J. Burguete and M. A. Miranda, Magnetohydrodynamics 48, 69 (2012).

[16] R. Moreau, Magnetohydrodynamics (Kluwer Academic Publishers, Dordrecht, The Netherlands, 1990).
[17] P. M. Glover, I. Cavin, W. Qian, R. Bowtell, and P. A. Gowland, Bioelectromagentics 28, 349 (2007).

[18] O. Patzold, K. Niemietz, R. Lantzsch, V. Galindo, I. Grants, M. Bellmann, and G. Gerbeth, Eur. Phys. J. Special Topics 220, 243 (2013).

[19] L. Feng and W. Y. Shi, Int. J. Heat Mass Transf. 101, 629 (2016).

[20] S. Smolentsev, N. B. Morley, M. A. Abdou, and S. Malang, Fusion Eng. Des. 100, 44 (2015).

[21] L. Marie, J. Burguete, F. Daviaud, and J. Leorat, Eur. J. Phys. B 33, 469 (2003).

[22] N. Plihon, G. Bousselin, F. Palermo, J. Morales, W. J. T. Bos, F. Godeferd, M. Bourgoin, J. F. Pinton, M. Moulin, and A. Aanesland, J. Plasma Phys. 81, 345810102 (2015).

[23] S. Olvera, E. M. A. Estrada, J. Sanchez-Marcos, F. J. Palomares, L. Vazquez, and P. Herrasti, Mater. Charact. 105, 136 (2015).

[24] D. C. Roberts, V. Marcelli, J. S. Gillen, J. P. Carey, C. C. Santina Della, and D. S. Zee, Curr. Biol. 21, 1635 (2011).

[25] N. B. Morley, J. Burris, L. C. Cadwallader, and M. D. Nornberg, Rev. Sci. Instrum. 79, 056107 (2008).

[26] K. Makimoto, T. Takeda, and H. Silverstein, Arch. Oto-RhinoLaryng. 220, 259 (1978).

[27] W. Merzkirch, Flow Visualization (Academic Press, New York, 1987).

[28] M. A. Miranda and J. Burguete, Phys. Rev. E 78, 046305 (2008); 79, 046201 (2009).

[29] See Supplemental Material at http://link.aps.org/supplemental/ 10.1103/PhysRevE.96.013103 for videos on the dynamical behavior of the patterns.

[30] B. Sreenivasan, P. Davidson, and J. Etay, Phys. Fluids 17, 117101 (2005).

[31] H. Ji, W. Fox, D. Pace, and H. L. Rappaport, Phys. Plasmas 12, 012102 (2005).

[32] G. Alcalá, M. Rivero, and S. Cuevas, Appl. Thermal Eng. 75, 1296 (2015).

[33] A. Giesecke, F. Stefani, and J. Burguete, Phys. Rev. E 86, 066303 (2012).

[34] J. Miles, J. Fluid Mech. 245, 485 (1992). 